\title{
Quorum-sensing regulates biofilm formation in Vibrio scophthalmi
}

\author{
Cristina García-Aljaro ${ }^{1 *}$, Silvia Melado-Rovira', Debra L Milton² and Anicet R Blanch
}

\begin{abstract}
Background: In a previous study, we demonstrated that Vibrio scophthalmi, the most abundant Vibrio species among the marine aerobic or facultatively anaerobic bacteria inhabiting the intestinal tract of healthy cultured turbot (Scophthalmus maximus), contains at least two quorum-sensing circuits involving two types of signal molecules (a 3-hydroxy-dodecanoyl-homoserine lactone and the universal autoinducer 2 encoded by luxS). The purpose of this study was to investigate the functions regulated by these quorum sensing circuits in this vibrio by constructing mutants for the genes involved in these circuits.

Results: The presence of a homologue to the Vibrio harveyi luxR gene encoding a main transcriptional regulator, whose expression is modulated by quorum-sensing signal molecules in other vibrios, was detected and sequenced. The $V$. scophthalmi LuxR protein displayed a maximum amino acid identity of $82 \%$ with SmcR, the LuxR homologue found in Vibrio vulnificus. luxR and luxS null mutants were constructed and their phenotype analysed. Both mutants displayed reduced biofilm formation in vitro as well as differences in membrane protein expression by mass-spectrometry analysis. Additionally, a recombinant strain of $V$. scophthalmi carrying the lactonase AiiA from Bacillus cereus, which causes hydrolysis of acyl homoserine lactones, was included in the study.

Conclusions: $V$. scophthalmi shares two quorum sensing circuits, including the main transcriptional regulator luxR, with some pathogenic vibrios such as $V$. harveyi and $V$. anguillarum. However, contrary to these pathogenic vibrios no virulence factors (such as protease production) were found to be quorum sensing regulated in this bacterium. Noteworthy, biofilm formation was altered in luxS and luxR mutants. In these mutants a different expression profile of membrane proteins were observed with respect to the wild type strain suggesting that quorum sensing could play a role in the regulation of the adhesion mechanisms of this bacterium.
\end{abstract}

Keywords: Vibrio scophthalmi, Biofilm formation, Quorum-sensing, AiiA, LuxS, Acyl homoserine lactone

\section{Background}

$V$. scophthalmi is the most abundant species among the marine aerobic or facultatively anaerobic bacteria present in the intestinal tract of cultured turbot (Scophthalmus maximus) even though it is not the most abundant Vibrio species in the surrounding water $[1,2]$. However, the possible benefits of turbot colonization by this bacterium are not well understood.

Bacteria communicate with members of their own species and even with bacteria outside of the species boundary to coordinate their behaviour in response to the density of the bacterial population, which is known as quorum-

\footnotetext{
*Correspondence: crgarcia@ub.edu

'Departament de Microbiologia, Facultat de Biologia, Universitat de Barcelona, Barcelona 08028, Spain

Full list of author information is available at the end of the article
}

sensing [3]. This communication relies on the production and sensing of one or more secreted low-molecular-mass signalling molecules, such as $\mathrm{N}$-acylhomoserine lactones (AHLs), the extracellular concentration of which is related to the population density of the producing organism. Once the signalling molecule has reached a critical concentration, the quorum-sensing regulon is activated and the bacteria elicit a particular response as a population.

The first quorum-sensing system identified was shown to control bioluminescence in Vibrio fischeri through the LuxI-LuxR system $[4,5]$. LuxI synthesizes a diffusible signal molecule, $\mathrm{N}$-(3-oxohexanoyl)-L-homoserine lactone (3-oxo-C6-HSL), which increases in concentration as the cell density increases. LuxR, the transcriptional activator of the bioluminescence lux operon, binds 3-oxo-C6HSL, which increases its stability. This complex binds

\section{() Biomed Central}


the promoter of the lux operon activating the production of light. The LuxI-LuxR quorum-sensing circuit is found in many Gram-negative bacteria and has been shown to regulate a variety of genes; for instance, it has been shown to regulate virulence in Pseudomonas aeruginosa [6]. However, this quorum-sensing circuit initially described in $V$. fischeri is not present in all Vibrio spp.

In Vibrio harveyi three additional quorum-sensing circuits were characterized that respond to three different signal molecules (see [7], for review). The first quorumsensing system is composed of an AHL synthase, LuxM, which is responsible for the synthesis of 3-hydroxy-C4HSL, and the receptor LuxN, a hybrid sensor kinase (present in V. harveyi, Vibrio anguillarum and Vibrio parahaemolyticus, among others). The second is composed of LuxS, LuxP and LuxQ. LuxS is responsible for the synthesis of the autoinducer 2 (AI-2), a universal signaling molecule used both by Gram-negative and Grampositive bacteria for interspecies communication [8], LuxP is a periplasmic protein that binds AI-2 and LuxQ is a hybrid sensor kinase. The third system is composed of CqsA and CqsS. CqsA is responsible for the synthesis of a different autoinducer, the cholerae autoinducer CAI-I [9], and CqsS is the hybrid sensor kinase. These three quorum-sensing systems converge via phosphorelay signal transduction to a single regulator $\mathrm{LuxO}$, which is activated upon phosphorylation at low cell density. LuxR, a regulatory protein that shares no homology to the $V$. fischeri LuxR, activates bioluminescence, biofilm formation, and metalloprotease and siderophore production at high cell density, is at the end of this cascade [10]. This regulatory protein is repressed at low cell density and derepressed at high cell density in the presence of autoinducers which, after binding, activate the phosphatase activity of the sensor kinases. This more complex quorum-sensing system is found predominately in Vibrio species and components of the network vary between species [7].

In a previous work, we demonstrated the presence of two quorum-sensing signal molecules in the supernatants of $V$. scophthalmi: $N$-(3-hydroxydodecanoyl)-L-homoserine lactone (3-hydroxy-C12-HSL) and AI-2, encoded by a luxS gene [11]. However, there is still a lack of knowledge of the bacterial activities that are regulated by quorumsensing in this bacterium. In this study, we identified a homologue of the $V$. harveyi luxR transcriptional regulator and analyzed the functions regulated by LuxR and the previously identified quorum-sensing signaling molecules by constructing mutants for the coding genes.

\section{Results and discussion}

\section{Detection and sequencing of luxR homologue}

In a previous study we demonstrated the presence of two quorum sensing signals in the supernatants of $V$. scophthalmi, a 3-hydroxy-C12-HSL and the AI-2 [11]. This fact suggested that $V$. scophthalmi could have two quorum-sensing circuits homologous to those identified in $V$. harveyi that converge in the luxR transcriptional regulator. In the present study the genome of $V$. scophthalmi A089 and A102 strains was screened by PCR analysis for the presence of luxR homologues using the primers listed in Table 1. For $l u x R$, a 636-bp fragment was generated and sequence analysis showed that this fragment shared high similarity to the $V$. harveyi-like $l u x R$ transcriptional regulator, which belongs to the TetR subfamily of transcriptional regulators [12]. The sequence of the complete $l u x R$ gene obtained by inverted PCR and showed a maximum nucleotide identity with $V$. parahaemolyticus (75\%) although the maximum amino acid identity and similarity was with $V$. vulnificus (82\% and $90 \%$, respectively) (Table 2). In addition, the 5'- and 3'-flanking DNA sequence of the $l u x R$ gene was also determined. The upstream region showed $87 \%$ identity with an intergenic region of $V$. tubiashii located between the hypoxanthine phosphoribosyltransferase $(h p t)$ gene and luxR [13]. The downstream region of the V. scophthalmi luxR gene contained an ORF that showed a maximum identity of $87 \%$ with the dihydrolipoamide dehydrogenase gene (lpd) of $V$. parahaemolyticus [14]. This genetic organization has also been described in some other vibrios such as $V$. cholerae and $V$. vulnificus [15], suggesting that they have been acquired by vertical transmission from a common ancestor.

\section{Functions regulated by luxR, luxS and AHLs}

In order to uncover the functions regulated by quorumsensing in $V$. scophthalmi null mutants for $l u x R$ and $l u x S$ were constructed. Additionally, a recombinant strain generated in a previous study that carries a gene coding for a lactonase from Bacillus cereus (AiiA) which was previously shown to hydrolyse AHLs [11] was included in the assays to study the functions regulated by AHLs.

No differences in growth rates were detected between the $\operatorname{luxR}$ and $\operatorname{luxS}$ mutants and the wild type strains. However, over-expression of $l u x R$ resulted in a decreased growth rate. The strains over-expressing $l u x R$ arrived to the stationary phase with a delay compared to the luxR mutant carrying the plasmid alone (Figure 1a). Similarly, although motility was not affected with statistical significance in $\operatorname{luxR}$ and $\operatorname{luxS}$ null mutants, over-expression of luxR caused about $50 \%$ decrease in motility in the swimming plate assay $\left(31.8 \mathrm{~mm}^{+} /-7.6 \mathrm{~mm}\right.$ in the strain over-expressing luxR and $54.3 \mathrm{~mm}^{+} /{ }_{-} 8.1$ in the control strain, after 24 hours), which is likely due to the decrease in the growth rate and not to downregulation of the genes involved in motility. The recombinant strain carrying the lactonase AiiA, had a much longer lag phase before reaching exponential growth which was then at a similar rate to that of the parent strain (Figure 1b) and 
Table 1 Primers used in this study

\begin{tabular}{|c|c|c|c|}
\hline & Sequence $\left(5^{\prime}-3^{\prime}\right)$ & Target gene & Reference \\
\hline LuxR-A & GGACTAGTTACTAATTAGGGCAA & luxR null mutant & This study \\
\hline LuxR-B & ATAAATACACAACATGAGTCGGGTGCGGGG & " & " \\
\hline LuxR-C & ATGTTGTGTATTTATAAAGAAGAA & " & $"$ \\
\hline LuxR-D & CTCGAGCTCGAGTCAGTGGGTCTA & " & " \\
\hline LuxR-G & CCGGAATTCCATTTGGCAAGGATT & Over expression luxR & $"$ \\
\hline LuxR-H & CGCGGATCCGGTGATGAGTITCAC & " & " \\
\hline LuxR-1 & CCATTACACTCATAAGCGCGA & Sequencing lux $R$ and & " \\
\hline LuxR-2 & TCGAGATGGGTTGTGACGCTG & flanking regions & “ \\
\hline LuxRI-F2 & GCACCATTACACTCAT & Detection of luxR & " \\
\hline LuxRI-R2 & TITGATGAACATGTITTG & " & " \\
\hline LuxRI-F4 & AAGTGTGGTTGAGTGGA & Detection of luxR and & " \\
\hline LuxRI-R4 & TAAGCAACAGCTGATGGA & flanking regions & " \\
\hline LuxS-F6 & CGATCTTGCTCTACCGGCT & Sequencing luxS & " \\
\hline LuxS-R7 & GAGTGCATCGCTGCAGTAC & flanking regions & " \\
\hline LuxS-A & GGACTAGTCTGGCTTATCACGAAG & luxs null mutant & " \\
\hline LuxS-B & CTCATTGAGCATTCGACAGTAAAGCTATC & " & " \\
\hline LuxS-C & GAAATGCTCAATGAGCTTCGCGTC & " & " \\
\hline LuxS-D & CTCGAGCTCGGACACTCGATCCACA & " & " \\
\hline LuxS-PMMBF & CCGGAATTCGCCAGCAGGAGAAGGACA & Over expression luxS & " \\
\hline LuxS-PMMBR & CGCGGATCCCGCTATCGATTAATCGA & " & " \\
\hline LuxS-Al & GGATCCGCCAGCAGGAGAAGGACA & Cloning of luxS into pACYC184 & " \\
\hline LuxS-BI & GTCGACCGCTATCGATTAATCGAC & & " \\
\hline
\end{tabular}

Restriction sites for Spel (ACTAGT), BamHI (GGATCC), EcoRI (GAATC), Sall (GTCGAC) and Sacl (GAGCT) are indicated in bold.

showed also a reduction about $50 \%$ of motility with respect to the control strain $\left(11.5 \mathrm{~mm}^{+} /{ }_{-} 3.3 \mathrm{~mm}\right.$ in the recombinant strain and $24.0 \mathrm{~mm}{ }^{+} /{ }_{-} 6.5 \mathrm{~mm}$ in the control strain). In the case of luxS over-expression no differences in the growth rate was observed for any of the strains.

In contrast, quorum-sensing was shown to positively regulate biofilm formation in vitro since both $\operatorname{luxR}$ and luxS null mutants had altered biofilm formation (Figure 2). Noticeably, biofilm was only formed when bacteria were grown in $\mathrm{MB}$ medium in either the mutant or the wildtype strains and abolished when bacteria were cultured in TSB2 (data not shown). MB medium is used to culture heterotrophic marine bacteria and mimics the marine salt concentration and, although TSB also allowed growth of the bacterium, for some reason the differences in salt concentration or in nutrient or carbohydrate contents exerted an effect on biofilm formation. In order to investigate a possible effect of catabolite repression, we supplemented MB with glucose $0.5 \%$ and $1 \% \mathrm{w} / \mathrm{v}$ which resulted in a decrease in biofilm formation. On the other hand, overexpression of luxR decreased the amount of biofilm, perhaps due to the decrease in the growth rate caused by the deregulation of $\operatorname{lux} R$, as stated above. In the case of
luxS overexpression no differences were found between the over-expressed $l u x S$ and the control strain carrying pMMB207 plasmid. Complementation of the A102 null luxS mutant strain with the pACYC184 plasmid reverted the strain to the wild type phenotype.

Positive and negative regulation of biofilm formation has been reported in other vibrio such as $V$. anguillarum and $V$. cholerae, respectively $[16,17]$. Interestingly, in a recent study on quorum-sensing in $V$. ichthyoenteri (the

Table 2 Percentage of nucleotide and amino acid identity and similarity of $V$. scophthalmi A089 LuxR with previously reported $\boldsymbol{V}$. harveyi-like LuxR regulators

\begin{tabular}{ll}
\hline Species & \% $\mathbf{n t}$ id (\% aa id/\% aa sim) \\
\hline V. alginolyticus (AF204737.1) & $74 \%(81 \% / 90 \%)$ \\
V. anguillarum (AF457643.2) & $73 \%(80 \% / 89 \%)$ \\
$V$. cholerae (EU523726.1) & $73 \%(76 \% / 87 \%)$ \\
$V$. harveyi (M55260.1) & $73 \%(79 \% / 90 \%)$ \\
$V$. mimicus (AB539839.1) & $71 \%(77 \% / 86 \%)$ \\
$V$. parahaemolyticus (AF035967.1) & $75 \%(80 \% / 90 \%)$ \\
$V$. vulnificus (EF596781.1) & $75 \%(82 \% / 90 \%)$ \\
\hline
\end{tabular}

GenBank Accession Number in brackets; nt, nucleotide; aa, amino acid; id, identity; sim, similarity. 

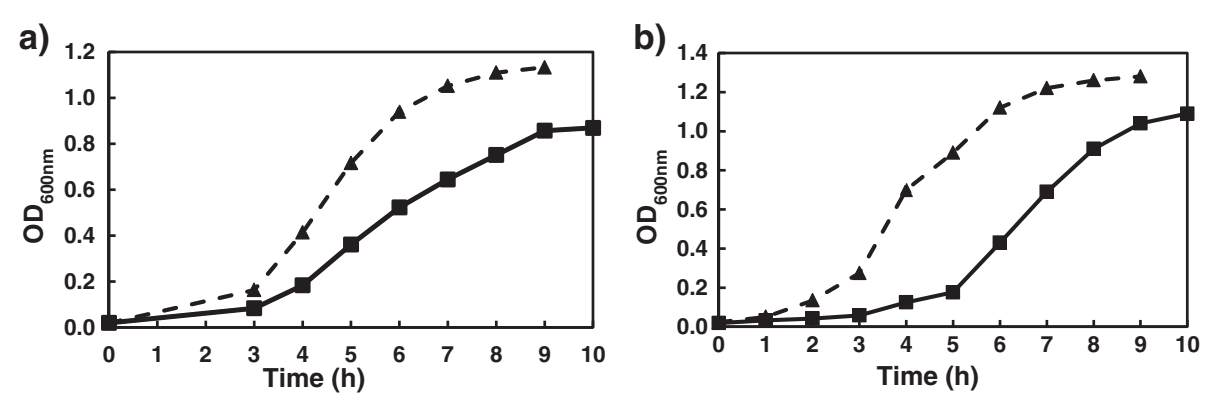

Figure 1 a) Effect of overexpression of luxR on the growth rate of V. scophthalmi. V. scophthalmi A089_23 (pMMB207) (black triangle) used as control strain vs V. scophthalmi A089_23 (pMMB207::IuxR) (black square); b) Effect of expression of the lactonase aiiA in V. scophthalmi A102. The growth rate was reduced in V. scophthalmi A089_23 overexpressing luxR (black square) compared to the control strain (black triangle) (Figure 1a), while strain A102_6.2 expressing the lactonase (black square) had a longer lag phase with respect to the control strain A102_pACYC (black triangle) (Figure 1b).

most closely related species to $V$. scophthalmi), its luxS homologue was sequenced and a mutant for this gene constructed, but no functions were reported to be regulated by this gene [18]. It has to be noted that neither the $V$. ichthyoenteri wild type, nor the luxS mutant formed biofilms in the microwell plates. Our results showed that $\operatorname{luxS}$ is involved in biofilm formation at least in vitro in $V$. scophthalmi. However, it is important to highlight that in our study the $V$. scophthalmi wildtype strain was only able to form significant biofilm when grown in MB, while TSB inhibited biofilm formation in vitro. Therefore, it would be interesting to assess if $V$. ichthyoenteri and the luxS mutant behave similarly to $V$. scophthalmi since they are so closely related. In $V$. scophthalmi, these two quorum-sensing systems may play a role in the colonization and establishment of this bacterium in the fish intestine, since it is a normal inhabitant of the turbot intestine [1]. In fact most vibrio species form biofilms on different structures, which is believed to be beneficial for the populations against different environmental stresses [19]. Work is currently being done to test these hypotheses.

A difference in the expression of membrane proteins, which may relate to differences in biofilm formation, was assessed by mass spectroscopy. In the case of the $\operatorname{luxS} \mathrm{mu}-$ tant the intensity of $\mathrm{m} / \mathrm{z} 4277$ was significantly lower than $\mathrm{m} / \mathrm{z} 4622$ and $\mathrm{m} / \mathrm{z} 4622$ was significantly higher than $\mathrm{m} / \mathrm{z}$ 5180 , while in the wild type strain these ratios were reversed $(p<0.01)$ (Figure 3$)$. Similar results were obtained for the luxR mutant, suggesting that the expression of these proteins were affected by these mutations.

Extracellular protease activity was not detected in either the wild-type strain or any of the $l u x R$ and/or $l u x S$ mutants as determined by a qualitative milk plate assay as well as a quantitative detection method using azocasein. On the other hand, siderophore production, which has been shown to be regulated by quorum-sensing in other vibrios was evaluated using the siderophore CAS assay. In addition, the ability to grow in iron depleted medium (EDDA assay) was assessed. A minor positive signal indicating the presence of siderophore activity was detected in all the mutants and wild type strains with the same intensity. However, neither the wild-type strain nor the mutants grew in the EDDA-supplemented medium suggesting that this species is not able to grow in irondepleted medium, at least under the conditions used in the assay. Extracellular proteases and siderophores are often produced by pathogenic vibrios [20-22], although some vibrios that are not pathogenic have been shown to produce siderophores in an iron-limited host environment, such as $V$. fischeri [23].

The Vibrio harveyi-like LuxR family of regulators is a diverse family with different associated functions depending on the Vibrio species. For example, in V. harveyi, luxR is expressed at high cell densities and regulates different functions including siderophores, colony morphology,

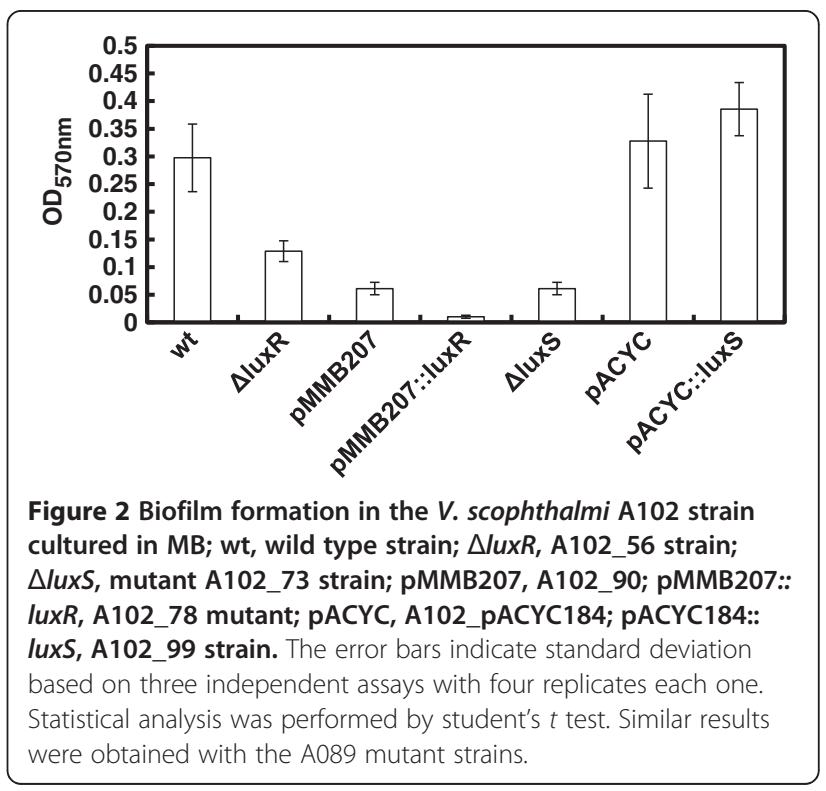



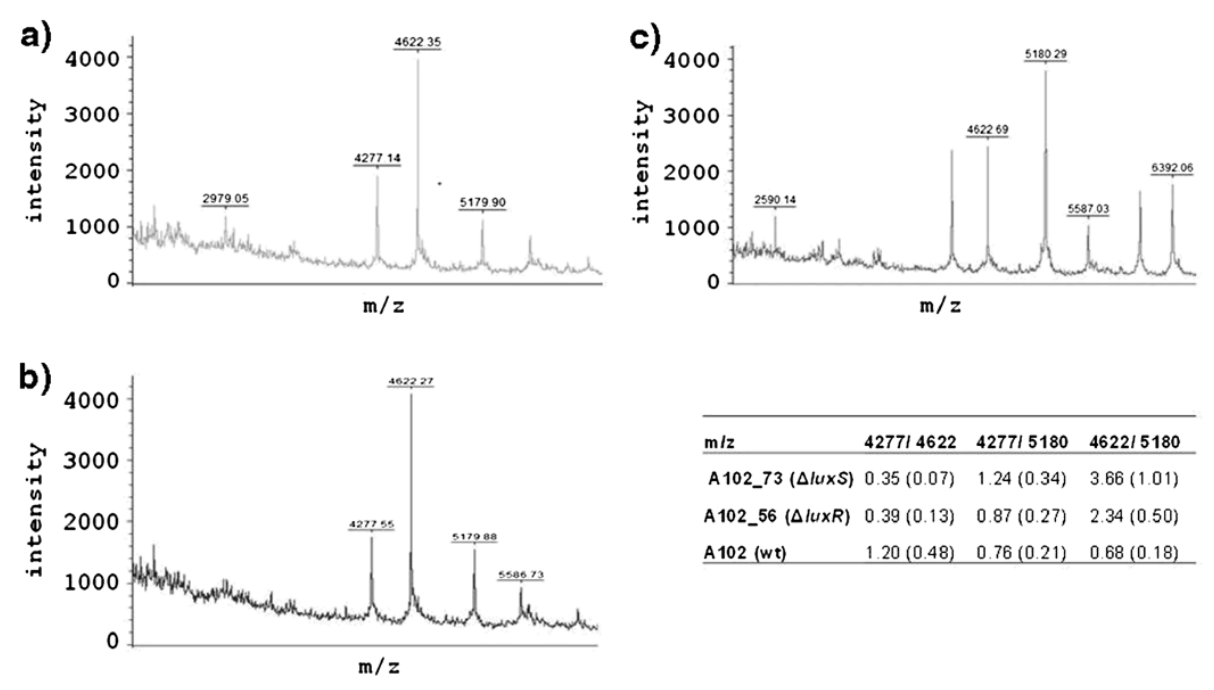

Figure 3 Differential expression of membrane proteins in the (a) V. scophthalmi A102 luxS and (b) luxR mutants with respect to the (c) wild type strain analyzed by mass spectrometry. The ratios of the major proteins with $\mathrm{m} / \mathrm{z} 4277,4622,5180$ are shown in the inset. Standard deviation of three independent measurements in brackets.

activates bioluminescence, activates metalloprotease production, represses the type III secretion system [21,24,25]. Apart from this diversity, all the $V$. harveyi-like quorumsensing systems converge to a phosphorelay circuit that regulates the expression of $l u x R$. However, in $V$. anguillarum, contrary to other members of the LuxR family, this gene is expressed at low densities. This gene represses exopolysaccharide production, and regulates biofilm formation, metalloprotease, pigment production and serine biosynthesis [17]. In the case of $V$. scophthalmi, which is a non-pathogenic vibrio, no virulence factors are shown to be regulated by this transcriptional regulator. At this moment, genome sequencing of the two $V$. scophthalmi strains used in this study is under process in our laboratory. Future work will involve transcriptome analysis of these mutants.

\section{Conclusions}

V. scophthalmi shares two quorum sensing circuits, including the main transcriptional regulator LuxR, with some pathogenic vibrios such as $V$. harveyi and $V$. anguillarum. However, contrary to these pathogenic vibrios no virulence factors (such as protease or siderophore production) were found to be quorum sensing regulated in this bacterium. Noteworthy, biofilm formation was altered in $\operatorname{luxS}$ and $\operatorname{luxR}$ mutants. In these mutants a different expression profile of membrane proteins were observed with respect to the wild type strain suggesting that quorum sensing could play a role in the adhesion and subsequent colonization of the fish by this bacterium. Further studies are needed in order to ascertain a similar behaviour of these mutants in vivo.

\section{Methods}

Bacterial strains, culture media and growth conditions

The bacterial strains and plasmids used in this study are listed in Table 3. The V. scophthalmi strains were grown at $30^{\circ} \mathrm{C}$ with agitation at $180 \mathrm{rpm}$ in either marine broth (MB, Difco) (filtered through a $0.1 \mu \mathrm{m}$ pore size to remove any precipitated salts that normally occur in this medium), or tryptic soy broth (TSB, Difco) supplemented with $\mathrm{NaCl}$ to a final concentration of $2 \%$ (TSB2). Luria Bertani (LB) broth was used for growth of Escherichia coli. When needed, antibiotics were added to the media at the following final concentrations: $5 \mu \mathrm{g} / \mathrm{ml}$ and $25 \mu \mathrm{g} / \mathrm{ml}$ chloramphenicol for $V$. scophthalmi and $E$. coli, respectively, and $100 \mu \mathrm{g} / \mathrm{ml}$ ampicillin for E. coli.

\section{Detection of luxR homologues by PCR}

Primers luxRI-F2 and luxRI-R2 (Table 1) were designed based on $V$. harveyi $\operatorname{luxR}$ and $l u x R$ homologue sequences from other vibrios retrieved from GenBank. Genomic DNA was used as template. Genomic DNA was isolated from single colonies by inoculating them in $20 \mu \mathrm{l}$ of double distilled $\mathrm{H}_{2} \mathrm{O}$ and boiling for $10 \mathrm{~min}$. The samples where then chilled and centrifuged for $5 \mathrm{~min}$ at 16,000 $g$ and $5 \mu \mathrm{l}$ of the supernatant was used as template for the PCR. The primers and reagents for PCR were purchased from Roche Diagnostics (Barcelona, Spain). The conditions used for the PCR are described elsewhere [26]. A 636-bp fragment containing part of the $\operatorname{luxR}$ gene was obtained.

\section{Cloning and sequencing of luxR gene and its flanking DNA}

The DNA sequence of the entire $\operatorname{lux} R$ gene of the two strains of $V$. scophthalmi together with the 5'- and 3'- 
Table 3 Bacterial strains and plasmids used in this study

\begin{tabular}{|c|c|c|}
\hline Strain or plasmid & Genotype and feature(s) & Reference \\
\hline \multicolumn{3}{|l|}{ V. scophthalmi strains } \\
\hline A089 & Wild type, turbot isolate (CECT 4638T) & [2] \\
\hline A102 & Wild type, turbot isolate (CECT 5965) & {$[1,2]$} \\
\hline A089_23 & A089 $\Delta /$ uxR mutant & This study \\
\hline A089_88 & A089_23 (pMMB207) & $"$ \\
\hline A089_75 & A089_23 (pMMB207::/uxR) mutant & $"$ \\
\hline A089_68 & A089 $\Delta /$ uxS mutant & $“$ \\
\hline A089_84 & A089_68 (pMMB207::/uxS) mutant & $"$ \\
\hline A089_92 & A089_68 (pMMB207) & $"$ \\
\hline A102_56 & A102 $\Delta /$ uxR mutant & $"$ \\
\hline A102_78 & A102_56 (pMMB207::/uxR) mutant & $"$ \\
\hline A102_90 & A102_56 (pMMB207) & “ \\
\hline A102_73 & A102 $\Delta /$ uxS mutant & $"$ \\
\hline A102_87 & A102_73 (pMMB207::/uxS) mutant & $“$ \\
\hline A102_94 & A102_73 (pMMB207) & $"$ \\
\hline A102_pACYC & A102 (pACYC184) & [11] \\
\hline A102_6.2 & A102 (pACYC184::aiiA) & $"$ \\
\hline A102_99 & A102_73 (pACYC:.:/uxS) & This study \\
\hline \multicolumn{3}{|l|}{ E. coli strains } \\
\hline $\mathrm{DH} 5 \mathrm{a}$ & E. coli used for transformation: $\lambda$ pir & Promega \\
\hline S17-1 & E. coli used for conjugation: $\lambda$ pir mob & {$[32]$} \\
\hline \multicolumn{3}{|l|}{ Plasmids } \\
\hline pDM4 & $\mathrm{Cm}^{r} \mathrm{Kan}^{r} \mathrm{SacBR}$; suicide vector & {$[28]$} \\
\hline pMMB207 & $\mathrm{Cm}^{\mathrm{r}}$ Ptac, broad host range expression vector & [33] \\
\hline pACYC184 & Tet $^{r}, \mathrm{Cm}^{r}$, broad host range expression vector & [34] \\
\hline pGEM T-easy & Ampr Kan'; TA cloning vector for sequencing & Promega \\
\hline
\end{tabular}

flanking regions was obtained by inverted PCR [27]. To prepare template for the inverted PCR, genomic DNA was digested with the restriction enzyme HincII and the linear HincII fragments were circularized by ligation with T4 DNA ligase (Invitrogen). The ligated DNA molecules were used as template to amplify a DNA fragment on which the 5'- and 3'-ends of the $\operatorname{luxR}$ gene have been joined at a HincII site. To amplify this fragment, primers (LuxRI-R4 and LuxRI-F4, Table 1) were designed to polymerize DNA out from either end of the 636-bp fragment that contains part of the $\operatorname{uxR}$ gene. A single amplimer was generated and sequenced to identify the flanking ends of the luxR gene. Using this sequence data, primers (LuxR-1 and LuxR-2, Table 1) were designed to amplify the entire $l u x R$ gene plus the 5'- and 3'-flanking DNA (a total of $944 \mathrm{bp}$ ). This fragment was cloned and sequenced using the LuxR-1 and LuxR-2 primers. These sequences were submitted to the GenBank database under the accession number JN684209 and JN684210, for V. scophthalmi A089 and A102, respectively.

\section{Sequencing of DNA that flanks the luxS gene}

The flanking regions of the previously sequenced luxS gene (accession number EF363481) were obtained as described above for $\operatorname{lux} R$, except that the restriction enzyme DraI and the primers LuxS-F6 and LuxS-R7 were used (Table 1).

\section{DNA sequencing}

DNA sequencing was performed with the Big Dye Terminator Cycle Sequencing Ready Reaction Kit 3.1 (Applied Biosystems), according to the manufacturer's instructions.

\section{Construction of $\Delta / u x R$ and $\Delta / u x S$ mutants by allelic exchange}

In-frame deletions of the $\operatorname{luxR}$ and $\operatorname{luxS}$ genes were generated by allelic exchange as previously described [28]. Briefly, an altered allele for both the $\operatorname{luxR}$ and the $\operatorname{luxS}$ genes was created by overlap PCR that encodes the first 12 amino acids fused to the last 9 amino acids, for $\operatorname{luxR}$ 
and the first 9 amino acids fused to the last 9 amino acids for luxS. The PCR primers LuxR-A, LuxR-B and LuxR-C, LuxR-D (Table 1) were used to create the luxR mutant allele and primers LuxS-A, LuxS-B, LuxS-C, and LuxS-D (Table 1) were used to create the luxS mutant allele. Both alleles were cloned into the R6K-origin based suicide vector pDM4 creating pDM4-luxR-AD and pDM4-luxS-AD, respectively. These plasmids were transferred to the V. scophthalmi A089 and A102 parental strains by bacterial conjugation as stated below, generating the $V$. scophthalmi A089_23 and A102_56 mutant, which carry a $l u x R$ in-frame deletion, and the $V$. scophthalmi A089_68 and A102_73 mutants, which carry a $l u x S$ in-frame deletion.

\section{Construction of mutants over-expressing luxR and luxS genes}

In order to determine the effect of over-expressing the $\operatorname{luxR}$ gene, the $\operatorname{luxR}$ and $\operatorname{luxS}$ genes were cloned into pMMB207 and fused to the tac promoter, which was induced using $0.5 \mathrm{mM}$ IPTG. To clone into this vector, primers LuxR-G and LuxR-H were used for $\operatorname{luxR}$ and LuxS-PMMBF and LuxS-PMMBR for luxS. In order to tranfer the pMMB207 plasmid alone or the pMMB207 plasmid carrying the $l u x S$ or $\operatorname{luxR}$ genes to $V$. scophthalmi luxR and luxS null mutants, the plasmid constructions were electroporated into E. coli S17-1. The plasmids were later transferred to $V$. scophthalmi by bacterial conjugation as stated below.

\section{Complementation of luxS null mutant}

Complementation of the A102_73 luxS mutant was performed by amplification of luxS gene with primers LuxS-AI and LuxS-BI (Table 1), followed by digestion with $\mathrm{Bam} H I$ and $\mathrm{Sal} I$ and ligation to the $\mathrm{pACYC}$ plasmid digested with the same strains (Table 3). The pACYC plasmid carrying the $\operatorname{luxS}$ gene was then electroporated into E. coli S17-1 (Table 3) and the transformants selected using $20 \mu \mathrm{g} / \mathrm{ml}$ chloramphenicol LB plates. This plasmid was later transferred to $V$. scophthalmi by bacterial conjugation and selected in TCBS with $5 \mu \mathrm{g} / \mathrm{ml}$ as stated below.

\section{Bacterial conjugation}

Plasmids pMMB207, pMMB207::luxR, pMMB207::luxS and pACYC:: luxS cloned into E. coli S17-1 were mobilized into $V$. scophthalmi by bacterial conjugation. Briefly, the E. coli S17-1 carrying the corresponding plasmid and the $V$. scophthalmi receptor strain were grown to midlogarithmic growth phase. A total of $0.5 \mathrm{ml}$ of the E. coli culture was pelleted in a microfuge, the supernatant was removed, and the cells were mixed with $1 \mathrm{ml}$ of $V$. scophthalmi. The cell mixture was centrifuged and suspended in $50 \mu \mathrm{l}$ of TSB2. The $50 \mu \mathrm{l}$ were spotted onto a
TSA2 plate and incubated at $30^{\circ} \mathrm{C}$ for $24 \mathrm{~h}$. Following incubation, the bacterial cells were resuspended in TSB2 and serial dilutions were plated onto TCBS medium (Oxoid) containing $5 \mu \mathrm{g} / \mathrm{ml}$ chloramphenicol to select for the $V$. scophthalmi containing the plasmids.

In order to construct the $V$. scophthalmi luxR and $\operatorname{luxS}$ null mutants, the E. coli S17-1 strains carrying either pDM4-luxR-AD and pDM4-luxS-AD were mated with $V$. scophthalmi A089 and A102 wild type strains. The selection for the strains carrying the suicide plasmid was performed in TCBS containing $5 \mu \mathrm{g} / \mathrm{ml}$ chloramphenicol as stated above. Afterwards, the null mutants were further selected after induction of sacBR in TSB2 agar plates supplemented with $5 \%$ sucrose. The in-frame deletions were confirmed by sequencing a PCR-amplified DNA fragment containing each mutation.

\section{Phenotypic assays \\ Growth rate}

The effect of the mutations on the growth rate of these bacteria was analysed. Briefly, ON cultures were prepared on TSB2 and diluted to an initial density of approximately 0.01 and incubated for $10 \mathrm{~h}$ at $30^{\circ} \mathrm{C}$ with continuous agitation. Bacterial growth was estimated from OD readings at $600 \mathrm{~nm}$ taken at different intervals.

\section{Protease activity}

Extracellular protease activity was evaluated both qualitatively and quantitatively. For qualitative assay the parental as well as the mutant strains were streaked onto TSA2 and MA supplemented with $1 \%, 1.5 \%$ or $2 \%$ skimmed milk and incubated for a maximum of $48 \mathrm{~h}$. The presence of a casein degradation halus was considered a positive result. The quantitative assay was performed as previously described using the azocasein assay as previously described [29], using $\mathrm{O} / \mathrm{N}$ supernatants of the strains to be tested.

\section{Biofilm formation}

Biofilm formation was evaluated using 96-well polystyrene cell-culture treated microtiter plates after $48 \mathrm{~h}$ incubation using the crystal violet staining method, as previously described [30]. Briefly, $\mathrm{O} / \mathrm{N}$ cultures of the corresponding strain to be tested were diluted into fresh TSB2 or MB media to get approximately an optical density of $0.01 \mathrm{OD}_{600 \mathrm{~nm}}$ units. A total of $200 \mu \mathrm{l}$ were dispensed in each well and incubated statically in a wet chamber for $48 \mathrm{~h}$ at $30^{\circ} \mathrm{C}$. A minimum of four replicates in three independent assays were measured.

\section{Motility}

MA and TSA2 swimming plates containing $0.25 \%$ agar were used to assess the effect of LuxS and LuxR in motility. An overnight culture of the corresponding strain to be 
analysed was diluted 1:100 and a drop containing $10 \mu \mathrm{l}$ of the sample was inoculated in the middle of the plate and the movement of the strains was monitored up to $48 \mathrm{~h}$ by measuring the diameter reached by the bacteria.

\section{Detection of siderophores}

The chrome azure assay (CAS) was used to detect the production of siderophores in both the mutants and wild type strains, as described in [31] with minor modifications. Briefly, the nutrient medium used for the growth of the bacteria was TSA supplemented with $0.5 \% \mathrm{NaCl}$. Additionally, the ability of these strains to grow on iron depleted media was assessed using MA and TSA2 plates containing $0.2 \mathrm{mM}$ ethylenediamine di(o-hydroxyphenylacetic acid) (EDDA) chelating agent.

\section{Membrane protein profiling by mass spectrometry}

Membrane proteins from the mutants and wild type strains were extracted from $500 \mathrm{ml} \mathrm{ON}$ cultures. Briefly, the cultures were centrifuged for $10 \mathrm{~min}$ at $16,000 \mathrm{~g}$ and washed with PBS. The cells were suspended in $10 \mathrm{ml}$ Tris $50 \mathrm{mM} \mathrm{pH} 8.0$ and the suspension was frozen at $-80^{\circ} \mathrm{C}$. Successive rounds of freezing and thawing were performed. The suspension was then centrifuged for $2 \mathrm{~min}$ at $16,000 \mathrm{~g}$. The supernatant was centrifuged at $16,000 \mathrm{~g}$ for 1 hour at $4^{\circ} \mathrm{C}$ and the pellet enriched in membrane proteins was suspended in $10 \mu \mathrm{l}$ of $50 \%$ acetonitrile-2.5\% trifluoroacetic acid. One microliter of the supernatant was placed onto a spot of a ground steel plate and air dried at room temperature. Each sample was overlaid with $1 \mu \mathrm{l}$ of matrix solution (saturated solution of $\alpha$-cyno-4-hydroxy-cinnamic acid in $50 \%$ acetonitrile- $2.5 \%$ trifluoroacetic acid) and air dried at room temperature.

Measurements were performed on an Autoflex III MALDI-TOF/TOF mass spectrometer (Bruker Daltonics, Leipzig, Germany) equipped with a $200-\mathrm{Hz}$ Smartbeam laser. Spectra were recorded in the linear, positive mode at a laser frequency of $200 \mathrm{~Hz}$ within a mass range from 2,000 to $20,000 \mathrm{Da}$. The IS1 voltage was $20 \mathrm{kV}$, the IS2 voltage was maintained at $18.7 \mathrm{kV}$, the lens voltage was $6.50 \mathrm{kV}$, and the extraction delay time was $120 \mathrm{~ns}$.

For each spectrum approximately 500 shots from different positions of the target spot were collected and analyzed. The spectra were calibrated externally using the Bruker Bacterial Test Standard (Escherichia coli extract including the additional proteins RNase A and myoglobin). Calibration masses were as follows: RL29 3637.8 Da; RS32, 5096.8 Da; RS34, 5381.4 Da; RL33meth, 6255.4 Da; RL29, 7274.5 Da; RS19, 10300.1 Da; RNase A, 13683.2 Da; myoglobin, $16952.3 \mathrm{Da})$. The analyses were performed in triplicate.

\section{Competing interests}

The authors declare that they have no competing interests.

\section{Authors' contributions}

CGA participated in the design, acquisition of data and wrote the manuscript; SMR participated in the acquisition and analysis of data; DLM has participated in the design of the study and has helped writing the manuscript; ARB participated in the design of the study and revision of the manuscript. All authors have read and approved the final version of the manuscript.

\section{Acknowledgements}

We would like to thank Barbara Weber, Ramon Rosselló-Mora, Ana Cifuentes and Rosa Maria Gomila for the technical assistance. This work was supported by the FEMS research grant ES-SEM2010-1Garcia-Aljaro, the Xarxa de Referència en Biotecnologia (XRB) and the Government of Catalonia's research program 2009SGR1043.

\section{Author details}

${ }^{1}$ Departament de Microbiologia, Facultat de Biologia, Universitat de Barcelona, Barcelona 08028, Spain. ²Department of Molecular Biology, Umeå University, Umeå 90187, Sweden.

Received: 11 May 2012 Accepted: 23 November 2012

Published: 3 December 2012

\section{References}

1. Cerda-Cuellar M, Blanch AR: Determination of Vibrio scophthalmi and its phenotypic diversity in turbot larvae. Environ Microbiol 2004, 6(3):209-217.

2. Cerda-Cuellar M, Rossello-Mora RA, Lalucat J, Jofre J, Blanch A: Vibrio scophthalmi sp. nov., a new species from turbot (Scophthalmus maximus). Int J Syst Bacteriol 1997, 47(1):58-61.

3. Fuqua WC, Winans SC, Greenberg EP: Quorum sensing in bacteria: the LuxR-Luxl family of cell density-responsive transcriptional regulators. J Bacteriol 1994, 176(2):269-275.

4. Engebrecht J, Silverman M: Identification of genes and gene products necessary for bacterial bioluminescence. Proc Natl Acad Sci USA 1984, 81(13):4154-4158.

5. Nealson KH, Platt T, Hastings JW: Cellular control of the synthesis and activity of the bacterial luminescent system. J Bacterio/ 1970, 104(1):313-322.

6. Lerat E, Moran NA: The evolutionary history of quorum-sensing systems in bacteria. Mol Biol Evol 2004, 21(5):903-913.

7. Milton DL: Quorum sensing in vibrios: complexity for diversification. Int J Med Microbiol 2006, 296(2-3):61-71.

8. Chen X, Schauder S, Potier N, Van Dorsselaer A, Pelczer I, Bassler BL, Hughson FM: Structural identification of a bacterial quorum-sensing signal containing boron. Nature 2002, 415(6871):545-549.

9. Higgins DA, Pomianek ME, Kraml CM, Taylor RK, Semmelhack MF, Bassler BL: The major Vibrio cholerae autoinducer and its role in virulence factor production. Nature 2007, 450(7171):883-886.

10. Miller MB, Bassler BL: Quorum sensing in bacteria. Annu Rev Microbiol 2001, 55:165-199.

11. Garcia-Aljaro C, Eberl L, Riedel K, Blanch AR: Detection of quorum-sensingrelated molecules in Vibrio scophthalmi. BMC Microbiol 2008, 8:138.

12. Ramos JL, Martinez-Bueno M, Molina-Henares AJ, Teran W, Watanabe K, Zhang X, Gallegos MT, Brennan R, Tobes R: The TetR family of transcriptional repressors. Microbiol Mol Biol Rev 2005, 69(2):326-356.

13. Hasegawa $H$, Hase CC: TetR-type transcriptional regulator VtpR functions as a global regulator in Vibrio tubiashii. Appl Environ Microbiol 2009, 75:7602-7609.

14. McCarter LL: OpaR, a homolog of Vibrio harveyi LuxR, controls opacity of Vibrio parahaemolyticus. J Bacteriol 1998, 180(12):3166-3173.

15. McDougald D, Rice SA, Kjelleberg S: The marine pathogen Vibrio vulnificus encodes a putative homologue of the Vibrio harveyi regulatory gene, luxR: a genetic and phylogenetic comparison. Gene 2000, 248(1-2):213-221.

16. Kovacikova G, Skorupski K: Regulation of virulence gene expression in Vibrio cholerae by quorum sensing: HapR functions at the aphA promoter. Mol Microbiol 2002, 46(4):1135-1147.

17. Croxatto A, Chalker VJ, Lauritz J, Jass J, Hardman A, Williams P, Camara M, Milton DL: VanT, a homologue of Vibrio harveyi LuxR, regulates serine, 
metalloprotease, pigment, and biofilm production in Vibrio anguillarum. J Bacteriol 2002, 184(6):1617-1629.

18. Li X, Han Y, Yang Q, Zhang XH: Detection of quorum sensing signal molecules and mutation of luxS gene in Vibrio ichthyoenteri. Res Microbiol 2010, 161(1):51-57.

19. Schembri MA, Givskov M, Klemm P: An attractive surface: gram-negative bacterial biofilms. Sci STKE 2002, 2002(132):re6.

20. Zhu J, Miller MB, Vance RE, Dziejman M, Bassler BL, et al: Quorum-sensing regulators control virulence gene expression in Vibrio cholerae. Proc Natl Acad Sci USA 2002, 99:3129-3134.

21. Lilley BN, Bassler BL: Regulation of quorum sensing in Vibrio harveyi by LuxO and sigma-54. Mol Microbiol 2000, 36(4):940-954.

22. Wang Q, Liu Q, Ma Y, Rui H, Zhang Y: LuxO controls extracellular protease, haemolytic activities and siderophore production in fish pathogen Vibrio alginolyticus. J Appl Microbiol 2007, 103:1525-1534.

23. Graf J, Ruby EG: Novel effects of a transposon insertion in the Vibrio fischeri glnD gene: defects in iron uptake and symbiotic persistence in addition to nitrogen utilization. Mol Microbiol 2000, 37:168-179.

24. Mok KC, Wingreen NS, Bassler BL: Vibrio harveyi quorum sensing: a coincidence detector for two autoinducers controls gene expression. EMBO J 2003, 22(4):870-881.

25. Henke JM, Bassler BL: Quorum sensing regulates type III secretion in Vibrio harveyi and Vibrio parahaemolyticus.J Bacteriol 2004, 186(12):3794-3805.

26. Garcia-Aljaro C, Muniesa M, Jofre J, Blanch AR: Prevalence of the stx2 gene in coliform populations from aquatic environments. Appl Environ Microbiol 2004, 70(6):3535-3540.

27. Ochman H, Gerber AS, Hartl DL: Genetic applications of an inverse polymerase chain reaction. Genetics 1988, 120:621-623.

28. Milton DL, O'Toole R, Horstedt P, Wolf-Watz H: Flagellin A is essential for the virulence of Vibrio anguillarum. J Bacteriol 1996, 178(5):1310-1319.

29. Denkin SM, Nelson DR: Induction of protease activity in Vibrio anguillarum by gastrointestinal mucus. Appl Environ Microbiol 1999, 65(8):3555-3560

30. Stepanovic S, Vukovic D, Hola V, Di Bonaventura G, Djukic S, Cirkovic I, Ruzicka F: Quantification of biofilm in microtiter plates: overview of testing conditions and practical recommendations for assessment of biofilm production by staphylococci. APMIS 2007, 115(8):891-899.

31. Schwyn B, Neilands JB: Universal chemical assay for the detection and determination of siderophores. Anal Biochem 1987, 160(1):47-56.

32. Miller $\mathrm{VL}$, Mekalanos JJ: A novel suicide vector and its use in construction of insertion mutations: osmoregulation of outer membrane proteins and virulence determinants in Vibrio cholerae requires toxR. J Bacteriol 1988, 170(6):2575-2583.

33. Morales VM, Backman A, Bagdasarian M: A series of wide-host-range lowcopy-number vectors that allow direct screening for recombinants. Gene 1991, 97(1):39-47.

34. Rose RE: The nucleotide sequence of pACYC184. Nucleic Acids Res Microbiol 1988, 16:355.

doi:10.1186/1471-2180-12-287

Cite this article as: García-Aljaro et al:: Quorum-sensing regulates biofilm formation in Vibrio scophthalmi. BMC Microbiology 2012 12:287.

\section{Submit your next manuscript to BioMed Central and take full advantage of:}

- Convenient online submission

- Thorough peer review

- No space constraints or color figure charges

- Immediate publication on acceptance

- Inclusion in PubMed, CAS, Scopus and Google Scholar

- Research which is freely available for redistribution

Submit your manuscript at www.biomedcentral.com/submit
Ciomed Central 